\title{
GRK2-Deficiency Reduces Insulin Activation of ERK1/2 and Mitogenesis in Mouse Liver FL83B Cells
}

\author{
Gulnar Shahid and Tahir Hussain*
}

Department of Pharmacological and Pharmaceutical Sciences, University of Houston, Houston, TX 77204, USA

\begin{abstract}
We have reported that G protein coupled receptor kinase-2 (GRK2) negatively regulates insulin receptor signaling leading to glycogen synthesis in mouse liver derived FL83B cells. Since insulin is a potent cellular growth hormone, present study investigated the effect of GRK2 on insulin-stimulated MAP kinase pathway leading to mitogenesis. Specific GRK2 siRNA was used to knock-down ( $>90 \%)$ GRK2 in FL83B cells. Effect of insulin on ERK1/2 activation and $\left[{ }^{3} \mathrm{H}\right]$-methyl thymidine incorporation were determined in GRK2 siRNA-treated and control cells. Insulinstimulated ERK1/2 activation was attenuated in GRK2-deficient as compared to control cells. Basal and insulinstimulated $\left[{ }^{3} \mathrm{H}\right]$-methyl thymidine incorporation, a measure of mitogenesis, was lower in GRK2-deficient cells. The data suggest that GRK2 may have positive regulatory role in insulin-stimulated MAP kinase pathway and mitogenesis. The present study together with our earlier report on insulin-induced glycogen synthesis indicates a dual role of GRK2 in insulin receptor signaling/function.
\end{abstract}

\section{INTRODUCTION}

In addition to its primary role in the maintenance of glucose homeostasis, insulin also affects other cellular functions such as promoting protein synthesis, mitogenesis or cellular growth [1]. These insulin functions are initiated by its binding to insulin receptor. Insulin binding to the $\alpha$ subunit of insulin receptor causes auto-phosphorylation of the $\beta$-subunit [2] and thereby increases the tyrosine kinase activity of the receptor towards other protein substrates. This results in the tyrosine phosphorylation of a family of insulin receptor substrate (IRS) proteins including IRS1, IRS2 and Sarcoma ( $\mathrm{Src}$ ) homology containing transforming proteins (Shc). The IRS proteins serve as docking proteins for other intracellular proteins containing Scr homology-2 (SH2) domains such as phospho-inositol-3 kinase (PI3 kinase) and growth factor receptor-bound protein-2 (Grb2). The binding of these proteins to IRS transmits the signal downstream, leading to the metabolic and mitogenic effects of insulin [3]. The interaction of phosphorylated IRS1/2 with PI3 kinase leads to activation of protein kinase B (PKB), also known as Akt, producing many effects such as glucose transporter type 4 (GLUT4) translocation, protein synthesis and glycogen synthesis [4-6]. The mitogenic pathway is initiated by binding of Grb2 to Shc leading to the activation of mitogenactivated proteins (MAP) kinase pathway including extracellular- signal-regulating kinase-1/2 (ERK1/2). A number of cytosolic and nuclear proteins are phosphorylated by ERK $1 / 2$ resulting in cellular growth and differentiation $[7,8]$.

The insulin receptor also couples to the G-proteins, Gi and $\mathrm{Gq}[9,10]$ and has been shown to influence signaling

\footnotetext{
*Address correspondence to this author at the Department of Pharmacological and Pharmaceutical Sciences, University of Houston, 521 Science and Research Bldg 2, Houston, TX 77204, USA; Tel: 713-7431274; Fax: 713-743-1884; E-mail: thussain2@uh.edu
}

components, which otherwise are known to couple and mediate $\mathrm{G}$ protein coupled receptor (GPCR) functions. Recently, others and we [11, 12] have shown that insulin signaling is potentially regulated by GPCR kinase- 2 (GRK2), member of ser/thr kinases known to desensitize GPCRs. GRK2 also is known as $\beta$-adrenergic receptor kinase-1 (BARK-1 or $\beta$-ARK-1) and adrenergic receptor, $\beta$, kinase-1 (ADBRK1). We found that GRK2 knock-down by small-interfering RNA (siRNA) caused an increase in basal and insulin-induced glycogen synthesis, and prevented insulin-induced desensitization of the insulin receptors [12]. This suggested GRK2 as a negative regulator of metabolic arm of insulin signaling and potentially affecting insulin sensitivity towards glucose metabolism. There are several reports suggesting that insulin resistance does not equally affect all aspects of insulin signaling. For example, while the signaling via metabolic pathway is diminished, the mitogenic pathway remains intact and may even be overactive due to higher plasma insulin levels during insulin resistance [13, 14]. However, the mechanisms of this differential regulation of the biological responses to insulin are not understood. Since GRK2 is suggested as a novel regulator of insulinsignaling $[11,12]$ and necessary for growth [15], we hypothesized that GRK2 may have differential role in insulin-induced mitogenic response. Therefore, we studied the role of GRK2 in insulin-induced activation of ERK1/2 and mitogenesis in mouse liver FL83B cells. We found that cellular deficiency of GRK2 attenuated insulin-stimulated phosphorylation of ERK1/2 and insulin-induced $\left[{ }^{3} \mathrm{H}\right]-$ thymidine incorporation in the FL83B cells.

\section{EXPERIMENTAL PROCEDURES}

Materials: FL83B, mouse hepatocyte cell line was obtained from American Tissue Culture Collection (Manassas, VA). F-12 nutrient mixture, oligofectamine, Opti-MemI, DMEM, fetal bovine serum, and antibioticantimycotic were purchased from Invitrogen (Carlsbad, CA). 
Trypsin was purchased from JRH Biosciences (Lenexa, Kansas). GRK2 siRNA was purchased from Dharmacon Inc (Chicago, IL). Horseradish peroxidase conjugated secondary antibodies and enhanced chemiluminescence kit were obtained from Alpha Diagnostic Intl (San Antonio, TX). Anti-phospho-ERK1/2 and anti-ERK1/2 antibodies were purchased from Cell Signaling Technology (Danvers, MA). $\left[{ }^{3} \mathrm{H}\right]$-methyl-thymidine was purchased from Amersham (Piscataway, NJ). All other chemicals were purchased from Sigma Aldrich (St. Louis, MO).

Cell Culture: The FL83B cells, a cell line derived from mouse liver, were grown in F12 nutrient medium supplemented with $10 \%$ fetal calf serum and $1 \%$ antibioticantimycotic (penicillin G, streptomycin sulphate and amphotericin B) at $37^{\circ} \mathrm{C}$ and $5 \% \mathrm{CO}_{2}$. Experiments were performed on cells from passage 3-15.

Transfection of FL83B Cells with GRK-2 siRNA: The transfection was performed using 40-50\% confluent hepatocytes, as described earlier [12]. GRK-2 siRNA (5'- U CGA UGC ACA CUG AAG UCA dAdT-3') and oligofectamine $(0.6 \%)$ were diluted separately in OptiMem I for $10 \mathrm{~min}$. For control sequence, a non-targeting siRNA (5'UAG CGA CUA AAC ACA UCA AUU-3') that has at least four mismatches to any human, mouse or rat gene was used instead of GRK2 siRNA sequence. The cells were washed with phosphate buffered saline (PBS), and serum free media was added. The cells were incubated with GRK2 siRNA or control sequence siRNA $(2 \mu \mathrm{M})$ for 4 hours followed by the addition of the growth medium containing $10 \%$ normal serum and $1 \%$ antibiotic. The cells were collected after 48 hours. In earlier study [12], we have optimized the GRK2 siRNA concentration and the transfection period in FL83B cells. Western blot analysis was performed using anti-GRK2 antibody to determine the levels of GRK2 in the cells. $\beta$ actin was labeled as the loading control.

Phosphorylation of ERK1/2: The control and GRK2 siRNA-treated cells were incubated without and with insulin $(100 \mathrm{nM})$ for 5 and $10 \mathrm{~min}$. Following insulin treatment, the phosphorylation of the ERK1/2 was measured by Western blot analysis using specific antibodies. Briefly, the hepatocytes (control and siRNA treated) were collected in lysis buffer containing protease inhibitors. Proteins in cell lysates were measured by BCA method using a kit (Pierce). Ten microgram of sample proteins from control and GRK2deficient cells were resolved by $10 \%$ SDS-PAGE and blotted on immobilon P membrane. Monoclonal anti-phospho-ERK$1 / 2$ antibody (1:1000) was used as the primary antibody and horseradish peroxidase-conjugated anti-mouse IgG (1:2000) was used as the secondary antibody. The bands were detected by enhanced chemiluminescence. The membranes were stripped and reprobed with polyclonal anti-ERK-1/2 antibody (1:1000). Ratio of the densitomeric values of phospho-ERK-1/2 and total ERK-1/2 was calculated and compared between control and siRNA treated cells.

$\left[{ }^{3} \mathbf{H}\right]-M e t h y l$ Thymidine Incorporation Assay: The hepatocytes were grown in 12 well plates and treated without (control) and with GRK2 siRNA for 48 hours, as described above. After transfection, the hepatocytes were starved overnight and subsequently treated with insulin for 12 hours followed by addition of $\left[{ }^{3} \mathrm{H}\right]$-methyl thymidine $(1 \mu \mathrm{Ci} /$ well $)$ to the wells. After 2 hours of incubation, the $\left[{ }^{3} \mathrm{H}\right]$-methyl thymidine incorporation was stopped by removal of the media and washing of the cells with ice-cold PBS. The cells were treated three times with $10 \%$ trichloro acetic acid for 10 $\min$ at $4^{\circ} \mathrm{C}$. The cells were dissolved in $\mathrm{HCl}(0.1 \mathrm{~N}, 300 \mu \mathrm{l})$ for $30 \mathrm{~min}$ at $37^{\circ} \mathrm{C}$. A $30 \mu \mathrm{l}$ sample was withdrawn for protein estimation. Rest of the sample was transferred to the liquid scintillation fluid for determining the radioactivity by Beckman scintillation counter. The sample radioactivity counts were normalized with sample protein. The data are presented as percent of control untreated cells.

\section{RESULTS}

Effect of GRK2 siRNA on GRK2 Expression in Liver FL83B Cells: Similar to our earlier studies [12], GRK2 siRNA treatment caused a reduction in GRK2 expression by $>90 \%$ compared with vehicle treated cells (Fig. 1). On the other hand, scrambled sequence siRNA had no affect on GRK2 expression, suggesting the specificity of the siRNA. Also, we have shown earlier that expression of GRK3, another member of GRK2 subfamily, is not affected by GRK2 siRNA treatment [12].

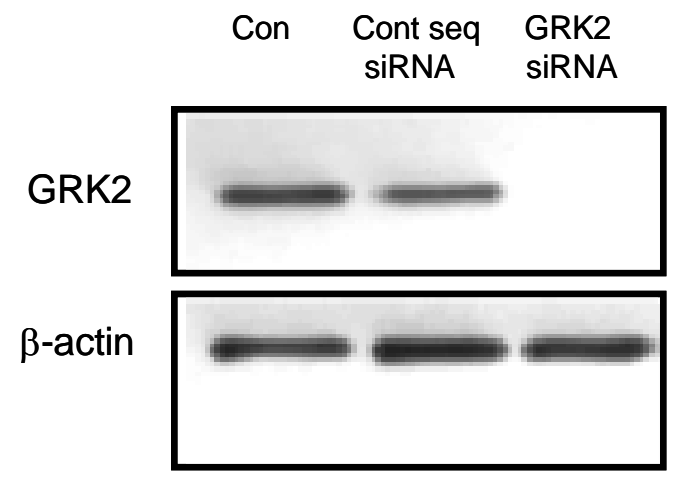

Fig. (1). Effect of GRK2 siRNA and control sequence siRNA on GRK2 expression in hepatocytes. Hepatocytes were transfected with $2 \mu \mathrm{M}$ of GRK2 siRNA or scrambled sequence siRNA. After 48 hours of transfection, cells were collected in lysis buffer and 4 $\mu \mathrm{g}$ of protein from each sample was subjected to western blotting using monoclonal GRK2 antibody. $\beta$-actin was labeled as loading control.

Effect of GRK2-Deficiency on Insulin-Stimulated Phosphorylation of ERK1/2: The phosphorylated-ERK1 $(\sim 44 \mathrm{kDa})$ and phosphorylated-ERK2 $(\sim 42 \mathrm{kDa})$ were determined by using anti-ERK1/2 antibody. This antibody detects dual phosphorylation at thr and tyr residues in thr-Xtyr motif of ERK1 and ERK2. This dual phosphorylation is required for the activity of ERK1 and ERK2. The insulin treatment increased the phosphorylation of ERK1 and ERK2 in control as well as in GRK2-deficient hepatocytes. The increase in ERK1 phosphorylation at $10 \mathrm{~min}$ was lower $(\mathrm{p}<0.05)$ in GRK2-deficient hepatocytes as compared to the control group. However, the increase in insulin-induced phosphorylation of ERK 1 was $16 \%$ and $67 \%$ (at 5 and 10 min) in control vs $9 \%$ and $37 \%$ in GRK2-deficient cells (Fig. 2A). Similarly, the insulin-induced phosphorylation of ERK2 in GRK2-deficient hepatocytes was lower $(p<0.05)$ as compared to control cells. The increase in phosphorylation of ERK2 was $87 \%$ and $131 \%$ (at 5 and $10 \mathrm{~min}$ ) in control $v s$ $50 \%$ and $85 \%$ (at 5 and $10 \mathrm{~min}$ ) in GRK2-deficient cells 
(Fig. 2B). The phosphorylation of ERK1/2 in un-stimulated cells (basal) was almost undetectable in both control and GRK2 deficient cells. The GRK2-deficiency did not affect the expression of ERK1/2 (Fig. 2A, B).

Effect of GRK2-Deficiency on Insulin-Stimulated Mitogenesis: The mitogenesis was measured as a function of $\left[{ }^{3} \mathrm{H}\right]$-methyl thymidine incorporation in DNA of the hepatocytes (Fig. 3). All the values are calculated as percent of the control basal. In control group, the insulin treatment increased $\left[{ }^{3} \mathrm{H}\right]$-methyl thymidine incorporation by $90 \%$ as compared to the control basal. Basal $\left[{ }^{3} \mathrm{H}\right]$-methyl thymidine incorporation in GRK2-deficient hepatocytes (46\%) was lower $(p<0.05)$ than the control basal. The insulin treatment increased the $\left[{ }^{3} \mathrm{H}\right]$-methyl thymidine incorporation in GRK2deficient hepatocytes but it was lower $(p<0.05)$ than the control cells. However, a comparison with respective basal values suggests that insulin-induced $\left[{ }^{3} \mathrm{H}\right]$ methyl thymidine incorporation was increased to similar extent $(\sim 2$-fold $)$ in both the control and GRK2-deficient cells. However, the reduction in the absolute activation of $\left[{ }^{3} \mathrm{H}\right]$ methyl thymidine incorporation in response to insulin suggests the potential limitation in the signaling along the MAPK kinase pathway in GRK2-deficient cells.

\section{DISCUSSION}

Earlier we reported a novel role of GRK2 as a negative regulator of insulin signaling along the metabolic pathway affecting glycogen synthesis in the liver cells. In the present study, we examined the role of GRK2 in insulin activation of mitogenic pathway. Contrary to the effects on glycogen synthesis, the cellular GRK2-deficiency caused a decrease in insulin-induced activation of ERK1/2; and this decrease was associated with attenuated mitogenic response to insulin.

Insulin signaling is a complex array of molecular events, which upon activation by insulin lead to a variety of cellular responses such as fuel metabolism, cellular growth etc. Numerous studies suggest the response selectivity of insulinsignaling pathways [1]. However, the regulatory
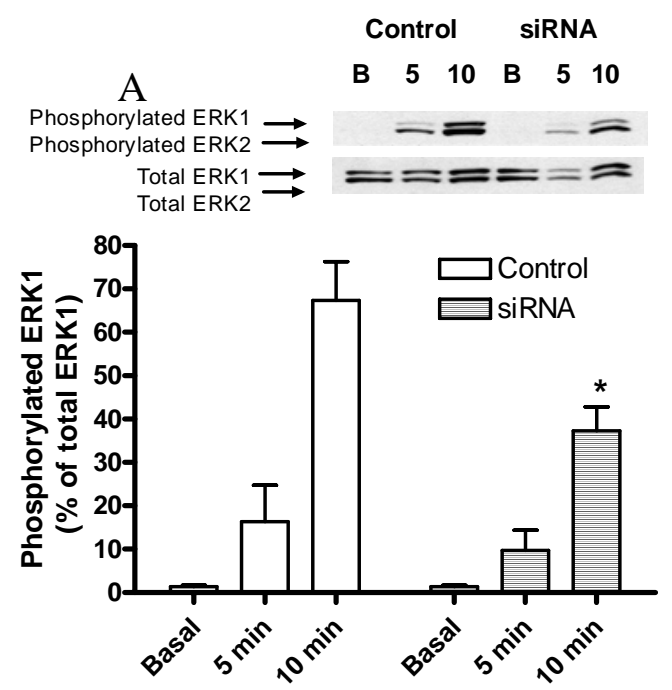

mechanism(s) that may be involved in sorting the diverse insulin signaling pathways and hence functions is not understood. Numerous reports suggest that insulin receptors not only belong to the family of tyrosine kinase receptor, but insulin receptors also are coupled to $G$ proteins $[9,10]$, suggesting a potential role of the $G$ protein associated signaling molecules in the regulation of insulin receptor function. GRK2 is a cytosolic protein with ser/thr kinase activity implicated in desensitization of a number of GPCR $[16,17]$. Recently, we suggested that GRK2 negatively regulates insulin-induced glycogen synthesis and may contribute to insulin receptor desensitization [12]. Similar findings were reported in fat cells showing GRK2 as an endogenous negative regulator of insulin signaling and insulin-induced cellular transport of glucose [11].

In addition to the metabolic functions associated with insulin, this peptide also serves as a cellular growth hormone and promotes mitogenesis via MAP kinase pathway. To investigate the role of GRK2 in insulin induced mitogenic pathway, we measured the phosphorylation of ERK1 and ERK2, a measure of MAP kinase pathway activation. It has been shown that MAP kinase in a negative feedback regulation phosphorylates GRK2 on $\operatorname{Ser}^{670}$ residue and decreases its activity, which in turn will prevent GPCR desensitization [18]. In our study, we found that GRK2-deficiency significantly reduced insulin-induced phosphorylation of ERK1/2 implicating GRK2 as a potential positive regulator of this pathway. This notion is supported by the observation that absolute mitogenic response to insulin in terms of $\left[{ }^{3} \mathrm{H}\right]$-methyl thymidine incorporation was significantly reduced in GRK2deficient cells as compared to control cells. However, GRK2 deficiency also reduced the basal (un-stimulated) $\left[{ }^{3} \mathrm{H}\right]$-methyl thymidine incorporation per se, suggesting a role of GRK-2 in cellular growth [15], even though the expression of ERK1 and ERK2 was not changed in GRK2 deficient cells as compared to the control. Homozygous GRK2 $2^{-/-}$knockout is known to have lethal effects on embryonic development suggesting the role of GRK2 in growth. Since ERK1/2 activation is known to stimulate mitogenesis, a decrease in the phosphorylation/

\section{B}

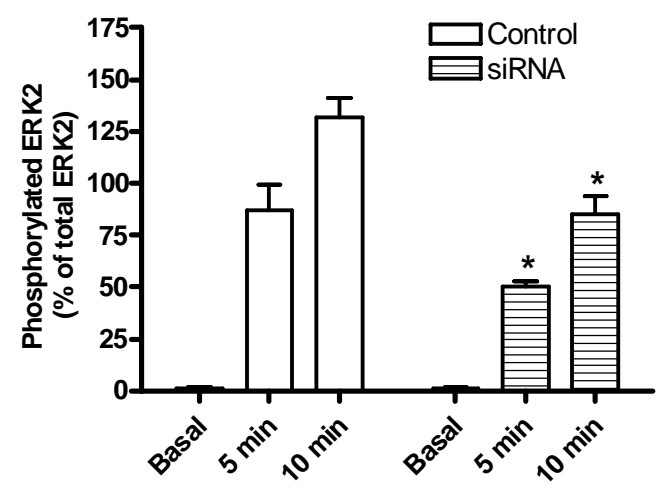

Fig. (2). Insulin-stimulated phosphorylation of ERK1 (A) and ERK2 (B) in control and GRK2 siRNA treated hepatocytes. Hepatocytes (control and siRNA treated) were incubated with insulin $(100 \mathrm{nM})$ for 5 and 10 min. ERK1/2 activation was determined by Western blot analysis using anti-phospho-ERK1/2 antibody and anti-ERK1/2. The bands were quantified by densitometric analysis. The values are presented as mean \pm S.E.M ( $\mathrm{n}=3$ ). *, compared with control (unpaired Student's t-test, $\mathrm{p}<0.05$ ). 
activation of ERK1/2 would result in decreased mitogenesis. Since $90 \%$ reduction in GRK2 by siRNA was not associated a parallel 90\% decrease in ERK1/2 phosphorylation in response to insulin, it is likely that another protein may be involved in mediating the interaction between ERK1/2 and GRK2.

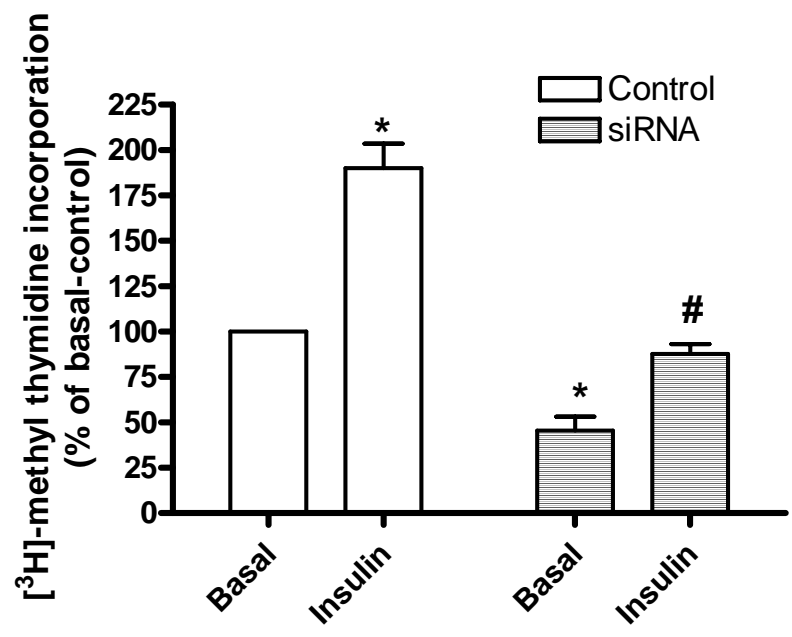

Fig. (3). Insulin-stimulated $\left[{ }^{3} \mathrm{H}\right]$-methyl thymidine incorporation in control and GRK2 siRNA treated hepatocytes. The hepatocytes (control and siRNA treated) were incubated with insulin $(100 \mathrm{nM})$ for 12 hours followed by incubation with $\left[{ }^{3} \mathrm{H}\right]$ methyl thymidine $(1 \mu \mathrm{Ci} /$ well $)$ for 2 hours. The radioactivity of $\left[{ }^{3} \mathrm{H}\right]$-methyl thymidine incorporated was measured by Beckman scintillation counter. Values, percent of control basal, are presented as mean \pm SEM, $\mathrm{n}=4$. *, control basal $v s$ siRNA basal and control basal vs control insulin. \#, control insulin $v s$ siRNA insulin (unpaired Student $\mathrm{t}$ test, $\mathrm{p}<0.05$ ).

The expression of GRK2 is increased in disease conditions such as cardiac failure, myocardial ischemia, and tissue specific inhibition of GRK2 in transgenic animals has led to enhanced contractility of heart muscles [19]. An enhanced GRK-2 expression has been reported in the kidney of obese Zucker rats, a model of insulin resistance and hyperinsulinemia [20]. It is not known whether GRK2 is up-regulated in other tissues, such as liver and muscles, and is responsible for the selective regulation of insulin receptor signaling. Based on our present data and earlier studies [11, 12], we would expect that up-regulated GRK2 would attenuate the metabolic arm, and increase the mitogenic arm of the insulin receptor signaling. Consistent with this notion, a decrease in insulin-mediated glucose metabolism without effecting insulin-mediated activation of MAP kinase pathway has been reported in aortic smooth muscles of obese Zucker rats $[13,14]$. The GRK2 is emerging as a common regulator for different receptor families, including insulin receptor $[11,12]$. Thus, changes in GRK2 expression/activity would affect not only a particular pathway but would change signaling along many other pathways. Understanding the mechanism of GRK2 regulated various signaling components/ pathways would help understand the diseases and the symptoms associated with them, especially insulin receptor-mediated functions in normal and insulin resistant animal models.

\section{REFERENCES}

[1] Saltiel AR, Pessin JE. Insulin signaling pathways in time and space. Trends Cell Biol 2002; 12: 65-71.

[2] Ullrich A, Bell JR, Chen EY, et al. Human insulin receptor and its relationship to the tyrosine kinase family of oncogenes. Nature 1985; 313: 756-61.

[3] Kahn CR, White MF. The insulin receptor and the molecular mechanism of insulin action. J Clin Invest 1988; 82: 1151-6.

[4] Avruch J. Insulin signal transduction through protein kinase cascades. Mol Cell Biochem 1998; 182: 31-48.

[5] Virkamäki A, Ueki K, Kahn CR. Protein-protein interaction in insulin signaling and the molecular mechanisms of insulin resistance. J Clin Invest 1999; 103: 931-43.

[6] Fang X, Yu SX, Lu Y, Bast RC Jr, Woodgett JR, Mills GB. Phosphorylation and inactivation of glycogen synthase kinase 3 by protein kinase A. Proc Natl Acad Sci USA 2000; 97: 11960-5.

[7] Boulton TG, Nye SH, Robbins DJ, et al. ERKs: a family of proteinserine/threonine kinases that are activated and tyrosine phosphorylated in response to insulin and NGF. Cell 1991; 65: 663-75.

[8] Morrison DK, Davis RJ. Regulation of MAP kinase signaling modules by scaffold proteins in mammals. Annu Rev Cell Dev Biol 2003; 19: 91-118.

[9] Krieger-Brauer HI, Medda PK, Kather H. Insulin-induced activation of NADPH-dependent $\mathrm{H} 2 \mathrm{O} 2$ generation in human adipocyte plasma membranes is mediated by Galphai2. J Biol Chem 1997; 272: 1013543.

[10] Imamura T, Huang J, Dalle S, et al. beta-Arrestin-mediated recruitment of the Src family kinase Yes mediates endothelin-1-stimulated glucose transport. J Biol Chem 2001; 276: 43663-7.

[11] Usui I, Imamura T, Satoh H, et al. GRK2 is an endogenous protein inhibitor of the insulin signaling pathway for glucose transport stimulation. EMBO J 2004; 23: 2821-9.

[12] Shahid G, Hussain T. GRK2 negatively regulates glycogen synthesis in mouse liver FL83B cells. J Biol Chem 2007; 282: 20612-20.

[13] Jiang ZY, Lin YW, Clemont A, et al. Characterization of selective resistance to insulin signaling in the vasculature of obese Zuker $(f a / f a)$ rats. J Clin Invest 1999; 104: 447-57.

[14] Cusi K, Maezono K, Osman A, et al. Insulin resistance differentially affects the PI 3-kinase- and MAP kinase-mediated signaling in human muscle. J Clin Invest 2000; 105: 311-20.

[15] Jaber M, Koch WJ, Rockman H, et al. Essential role of B-adrenergic receptor kinase 1 in cardiac development and function. Proc Natl Acad Sci USA 1996; 93: 12974-9.

[16] Krupnick JG, Benovic JL. The role of receptor kinases and arrestins in $\mathrm{G}$ protein-coupled receptor regulation. Annu Rev Pharmacol Toxicol 1998; 38: 289-319.

[17] Carman CV, Parent JL, Day PW, et al. Selective regulation of G $\alpha_{q^{\prime 11}}$ by an RGS domain in the G protein-coupled receptor kinase, GRK2. J Biol Chem 1999; 274: 34483-92.

[18] Pitcher JA, Tesmer JJ, Freeman JL, Capel WD, Stone WC, Lefkowitz RJ. Feedback inhibition of G protein-coupled receptor kinase 2 (GRK2) activity by extracellular signal-regulated kinases. J Biol Chem 1999; 274: $34531-4$.

[19] Iaccarino G, Koch WJ. Transgenic mice targeting the heart unveil G protein-coupled receptor kinases as therapeutic targets. Assay Drug Dev Technol 2003; 1: 347-55.

[20] Trivedi M, Lokhandwala MF. Rosiglitazone restores renal D1A receptor-Gs protein coupling by reducing receptor hyperphosphorylation in obese rats. Am J Physiol Renal Physiol 2005; 289: F298-304. 